\title{
Mechanical Study of PLA-PCL Fibers during In Vitro Degradation
}

\author{
A.C. Vieira ${ }^{1,2, *}$, J.C. Vieira ${ }^{1}$, J.M. Ferra ${ }^{3}$, F.D. Magalhães $^{3}$, R.M. Guedes ${ }^{2}$, A.T. Marques ${ }^{2}$ \\ ${ }^{1}$ UMEC, Institute of Mechanical Engineering and Industrial Management, \\ Rua Dr. Roberto Frias 378, 4200-465 Porto, Portugal \\ avieira@inegi.up.pt, jvieira@inegi.up.pt \\ ${ }^{2}$ DEMec, Mechanical Engineering Department, Faculty of Engineering of University of Porto (FEUP), \\ Rua Dr. Roberto Frias s/n, 4200-465 Porto, Portugal \\ rmguedes@fe.up.pt, marques@fe.up.pt \\ ${ }^{3}$ LEPAE - Chemical Engineering Department, Faculty of Engineering of University of Porto (FEUP), \\ Rua Dr. Roberto Frias s/n, 4200-465 Porto, Portugal \\ joao.ferra@fe.up.pt,fdmagalh@fe.up.pt
}

Keywords: PLA-PCL properties, Hydrolysis, Degradation, modeling

\begin{abstract}
The aliphatic polyesters are widely used in biomedical applications since they are susceptible to hydrolytic and/or enzymatic chain cleavage, leading to $\alpha$-hydroxyacids, generally metabolized in the human body. This is particularly useful for many biomedical applications, particularly, for temporary mechanical supports in regenerative medical devices. Ideally, the degradation should be compatible with the tissue recovering.

In this work, the mechanical properties evolution during degradation are discussed based on experimental data. The decrease of tensile strength of PLA-PCL fibers follows the same trend as the decrease of molecular weight, and so it can also be modeled using a first order equation. For each degradation stage, hyper elastic models such as neo-Hookean, MooneyRivlin and second reduced order, allowed a reasonable approximation of the material behavior. Based on this knowledge, constitutive models that describe the mechanical behavior during degradation are proposed and experimentally validated. The proposed theoretical models and methods may be adapted and used in other biodegradable materials, and can be considered fundamental tools in the design of regenerative medical devices where strain energy is an important requirement, such as ligaments, cartilage, stents or others.

\footnotetext{
* Corresponding author. Tel.: +35122 9578710; Fax: +351229537352.

e-mail address: avieira@inegi.up.pt (A.C. Vieira)
}

\section{Introduction}

PLA and PCL are two important members of the aliphatic polyesters class, among the biodegradable materials. For the purpose of an ongoing task, which consists on the 
dimensioning of a ligament augmentation device (LAD), we are studying models to simulate the mechanical behavior evolution with degradation which is relevant in the design phase of this biodegradable device. Both thermoplastics are hydrophilic with slow degradation rates, and for this reason they were selected for this particular application. Concerning PLA, it has been widely used since the 70's. There are two polyenantiomeric forms: poly (L-lactide) acid (PLLA) and poly(D-lactide) acid (PDLA). PDLA having a much higher degradation rate than PLLA. It degrades completely into lactic acid within a period ranging from 10 months to 4 years, depending upon its molecular weight, crystalline degree, material shape, and implantation site (Chen et al., 2003). PLLA is a rather less ductile and stiffer polymer with a low degradation rate. PLA has a modulus of elasticity about 3-4 GPa (Zuideveld et al., 2006; Van de Velde and Kiekens, 2002). It is often compounded with PCL, which has also a low degradation rate. Since PCL is very ductile and presents low stiffness, with modulus of elasticity around 0,21-0,44 GPa (Van de Velde and Kiekens, 2002), this blend improves the mechanical properties of original PLA (Saha and Tsuji, 2009). PLLA is glassy at body temperature (glass-transition temperature $\approx 60^{\circ} \mathrm{C}$ ), whereas PCL is rubbery (glass-transition temperature $\approx-60^{\circ} \mathrm{C}$ ). The elongation at break and strength of the compound are therefore improved in relation to pure PLLA. On the other hand, the degradation products of PLA are known to reduce local $\mathrm{pH}$, accelerate degradation and induce inflammatory reactions (Cheung et al., 2007). Blending it with PCL has the added benefit of minimizing local acidification, reducing the inflammatory response (Liao et al., 2008).

The degree of erosion associated to the biodegradation process is usually estimated from measurements of mass loss. The percentage of weight loss, WL\%, is computed from:

$W L \%=100\left(W_{0}-W_{r}\right) / W_{0}$

where $W_{0}$ and $W_{r}$ are, respectively, the initial and the residual weight of the specimen.

The erosion process can be described by phenomenological diffusion-reaction mechanisms. An aqueous media diffuses into the polymeric material while oligomeric products diffuse outwards. Within the polymeric matrix, hydrolytic reactions take place, mediated by water and/or enzymes.

Biomaterials can be classified into hydrolytically degradable polymers and enzymatically degradable polymers (Nair and Laurencin, 2007), according to the mechanism of covalent bond cleavage taking place. Most of the naturally occurring polymers, such us collagen or chitosan, among others, undergo enzymatic degradation. A list of enzymatically degradable polymers can be found in the work of Park et al. (1993). Their degradation rate varies significantly with the site of implantation, and among hosts, depending on the concentration of the enzymes.

Williams (1981) noticed that PLLA degradation rate was significantly affected by some enzymes, such as pronase, proteinase K and bromelain. Gan et al. (1999) reported the degradation of PCL in the blends of PLA-PCL in the presence of Pseudomonas lipase. Enzymes are large molecules unable to diffuse into the crystalline regions. So they promote surface erosion. A significant increase in the degradation rate was reported for PLLA during degradation in the presence of mixed culture of microorganisms compared to abiotic degradation (Hakkarainen et al., 2000). However, for most biodegradable materials, especially synthetic polymers, passive hydrolysis is the most important mode of degradation.

To model the erosion process in full, a complex mathematical model is needed to account for all the reaction steps and for the structural and morphologic details. The parameters in such a model require extensive experimentation. Modern numerical techniques have been used (Göpferich and Langer, 1993; Wang et al., 2008; Yu et al., 2008; Han and Pan, 2009; Bikiaris et al., 2007; Metzmacher et al., 2007) to solve the corresponding equations for 
devices of both simple and complicated geometries, in the context of drug release devices. However, none of these models were able to predict the mechanical properties evolution of the device.

Polymer degradation is the first step of erosion phenomenon and can be estimated by measuring the degradation of mechanical strength or the molecular weight decrease. The complete erosion of the polymer is known to take substantially more time than the loss of tensile strength. During this first phase aqueous solution penetrates the polymer, followed by hydrolytic degradation, converting this way long polymer chains into shorter water-soluble fragments, which can be regarded as a reverse polycondensation process. For example, PLA become soluble in water for molecular weight, Mn, below $\approx 20.000(\mathrm{~g} / \mathrm{mol})$ (Zhang et al., 2008).

The proposed constitutive models, which represent the mechanical behavior during degradation, are drawn after the relevant aspects of the phenomena that occur during degradation are described in detail, i.e. diffusion, hydrolytic damage, surface and bulk erosion. This is necessary to explain and justify the present theoretical assumptions. An experimental validation follows to prove that the tensile strength and the stress-strain plot evolutions during degradation can be determined for PLA-PCL fibers using the present approach. Finally, the limitations and the possible adaptations of these methods to develop more accurate models are also discussed.

\section{Diffusion}

The diffusion rate of water into the material can ideally be described by Fick's Second Law, presented below for one dimension (Crank, 1975), valid for isotropic polymers.

$\frac{d w}{d t}=D \frac{\partial^{2} w}{\partial x^{2}}$

where $w$ is the water concentration and $x$ is the position (in thickness or radius). The diffusion coefficient $D$ can be determined by inverse parameterization, measuring the increase in weight due to moisture absorption during incubation, on samples with two different diameters. The amount of absorbed water is computed from:

$m_{w}=100\left(m_{w_{s}}-m_{w_{r}}\right) / m_{w_{r}}$

where $m_{w r}$ and $m_{w s}$ are the weights of the specimen before and after swelling, respectively.

In the particular case of biodegradable polymers, water diffusion is very fast compared to water mediated hydrolysis. Therefore, water can be assumed, in many cases, uniformly distributed within the polymer from the beginning of erosion process, and hydrolysis promotes homogeneous bulk erosion (Li et al., 1990). As will be shown bellow, this can be assumed in the present case. This assumption is reasonable for small thickness devices, such as fibers.

\section{Hydrolytic Damage}

The polymeric ester groups can be easily hydrolyzed, leading to chain scission. Ester hydrolysis can be either acid or base catalyzed (Sykes, 1975). In figure 1 the acid based hydrolysis mechanism, more common in PLA degradation, is presented. A general consequence is the lowering of the plastic flow ability of the polymer, thus causing an increase in brittleness. 


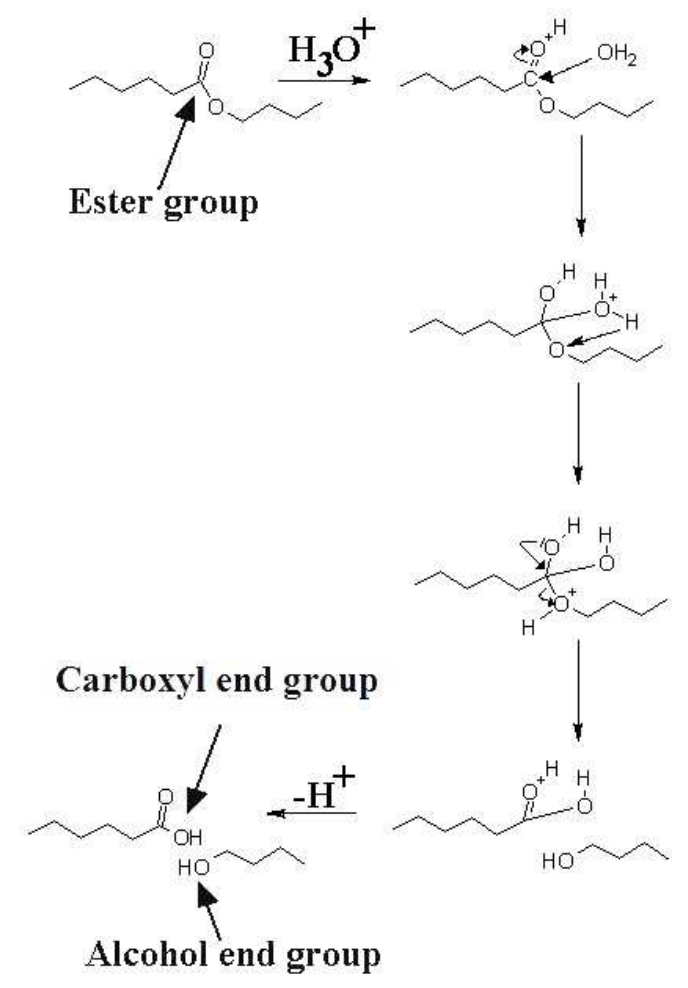

Figure 1 - Scheme of the acid based hydrolysis mechanism (Vieira et al., 2009)

Polyester hydrolysis has been traditionally modeled using a first order kinetic mechanism, following the Michaelis-Menten scheme (Bellenger et al., 1995). Each polymer molecule, with its own carboxylic and alcohol end groups, is broken in two, randomly in the middle at a given ester group. So, the number of carboxylic end groups will increase with degradation time, while the molecules are being split by hydrolysis. The following first-order equation describes the hydrolytic process (Farrar and Gilson, 2002), in terms of the rate of formation of carboxyl end groups:

$\frac{d C}{d t}=k E w C=u_{m} C$

where $E, C$ and $w$ are the concentrations of ester groups, carboxyl groups and water in the polymer matrix, respectively, $k$ is the hydrolysis kinetic constant and, $u_{m}$ is the hydrolysis rate, assuming that $E$ and $w$ are constant in the early stages of the reaction. The hydrolysis kinetic constant is a thermodynamic quantity associated to the probability of molecular scission, and it depends on temperature, load applied to the material and $\mathrm{pH}$ of the aqueous media. It is also assumed that water is uniformly distributed within the sample volume (no diffusion control). Since the concentration of carboxyl end groups is given by $C=1 / M_{n_{t}}$, where $M_{n}$ is the number-average molecular weight of the polymer, equation 4 becomes, after integration:

$$
M_{n_{t}}=M_{n_{0}} e^{-u_{m} t}
$$

It has been shown (Ward, 1983) that the fracture strength of a generic thermoplastic polymer can, in many cases, be related to $\mathrm{M}_{\mathrm{n}}$ through the empirical relationship: 
$\sigma=\sigma_{\infty}-\frac{B}{M_{n_{t}}}=\sigma_{\infty}-\frac{B}{M_{n_{0}} e^{-u_{m} t}}$

where $\sigma$ is the fracture strength, $\sigma_{\infty}$ is the fracture strength at infinite molecular weight, and $B$ is a constant. Equation 6 provides a description of the time dependence of the material's mechanical strength, which is relevant in the design phase of this biodegradable device. This is the only model that can be found in the literature, to simulate mechanical strength evolution (Farrar and Gilson, 2002). As this is an empirical equation, constant $B$ must be experimentally determined for each material.

We have further defined in (Vieira et al., 2009) damage due to hydrolysis as:

$$
d_{\mathrm{h}}=1-\frac{\sigma}{\sigma_{0}}
$$

For aliphatic polyesters, hydrolysis rates are affected by temperature, molecular structure, ester group density, type of degradation media and mechanical stimulus. The degree of cristallinity may be an additional factor of great importance, since the crystalline domains are less permeable to water penetration, slowing down hydrolysis. This way, material processing and storage conditions have a big influence on mechanical and degradation properties (Saha and Tsuji, 2009). The $\mathrm{pH}$ of the aqueous medium also affects the degradation reaction rates (Kirby, 1972). Tsuji et al. studied the hydrolysis of PLLA films at $37^{\circ} \mathrm{C}$ in alkaline solution (pH 12) (Tsuji and Ikada, 1998) acid solution ( $\mathrm{pH}$ 2.0) (Tsuji and Nakahara, 2001) and phosphate-buffered solutions ( $\mathrm{pH}$ 7.4)(Tsuji and Ikada, 2000). In the present case, $\mathrm{pH}$ can be considered constant. Also in the human body, $\mathrm{pH}$ is kept in homeostatic value. Temperature will increase diffusion due to increased molecular flexibility. This will also increase the degradation rate, due to the excitement of the molecules that will increase the probability of bond scissions. Also in the present case temperature is kept constant as in the human body, in the homeostatic value of around $37^{\circ} \mathrm{C}$. The influence of the mechanical environment in the degradation rate was also reported (Miller and Williams, 1984; Chu, 1985). It seems that loaded fibers degrade faster than unloaded ones, and the magnitude of degradation depend on the level of applied stress and the incubation time. Similarly to temperature, stress also increases the probability of bond scissions. In the present case no load was applied during degradation, and that's why hydrolysis kinetic constant $k$ is considered constant. But in most of the applications the material is submitted to a stress sate. When the stress state remains constant during degradation, degradation rate must be determined for that particular load case. If any variation occurs on the stress state, or the temperature, or on the environment, $k$ would be no longer constant.

The degradation occurs faster in the amorphous region. This fact explains the percentage increase of the crystalline phase. To model this phenomenon, knowing the initial crystalline degree, two different rates should be considered for both phases, and two different hydrolytic damage values should be calculated and added according to volume fractions.

\section{Surface vs Bulk Erosion}

Different types of erosion are illustrated in figure 2. One is homogeneous or bulk erosion without autocatalysis (figure $2 \mathrm{c}$ ), in which hydrolysis occurs simultaneously throughout the entire specimen. In this case diffusion is considered to occur instantaneously. Hence, the 
decrease in molecular weight, the reduction in mechanical properties, and the loss of mass also occur simultaneously throughout the entire specimen. For these cases the mechanical strength evolution and damage can be modeled using equations 6 and 7 and ignoring diffusion. One other type is heterogeneous or surface erosion (figure 2 a), in which hydrolysis occurs in the region near the surface, whereas the bulk material is only slightly or not hydrolyzed at all. As the surface is eroded and removed, the hydrolysis front moves through the material core. In this case, in which diffusion is very slow compared to hydrolysis, one must use equation 2 to calculate water concentration $w(t, x)$ at any instant $t$ through the thickness $\mathrm{x}$, before using equations 6 and 7. Surface eroding polymers have a greater ability to achieve zero-order release kinetics, and are therefore ideal candidates for developing drug delivery devices (Nair and Laurencin, 2007). Also enzymatic erosion fits on this last type of erosion, since enzymes are unable to diffuse and present a raised hydrolysis kinetic constant $k$. In the presence of enzymes, heterogeneous hydrolytic damage can be modeled considering a high hydrolysis kinetic constant $k$ and a diffusion coefficient $D$ close to zero. This damage should then be added to the damage due to water, either homogenous or heterogeneous.

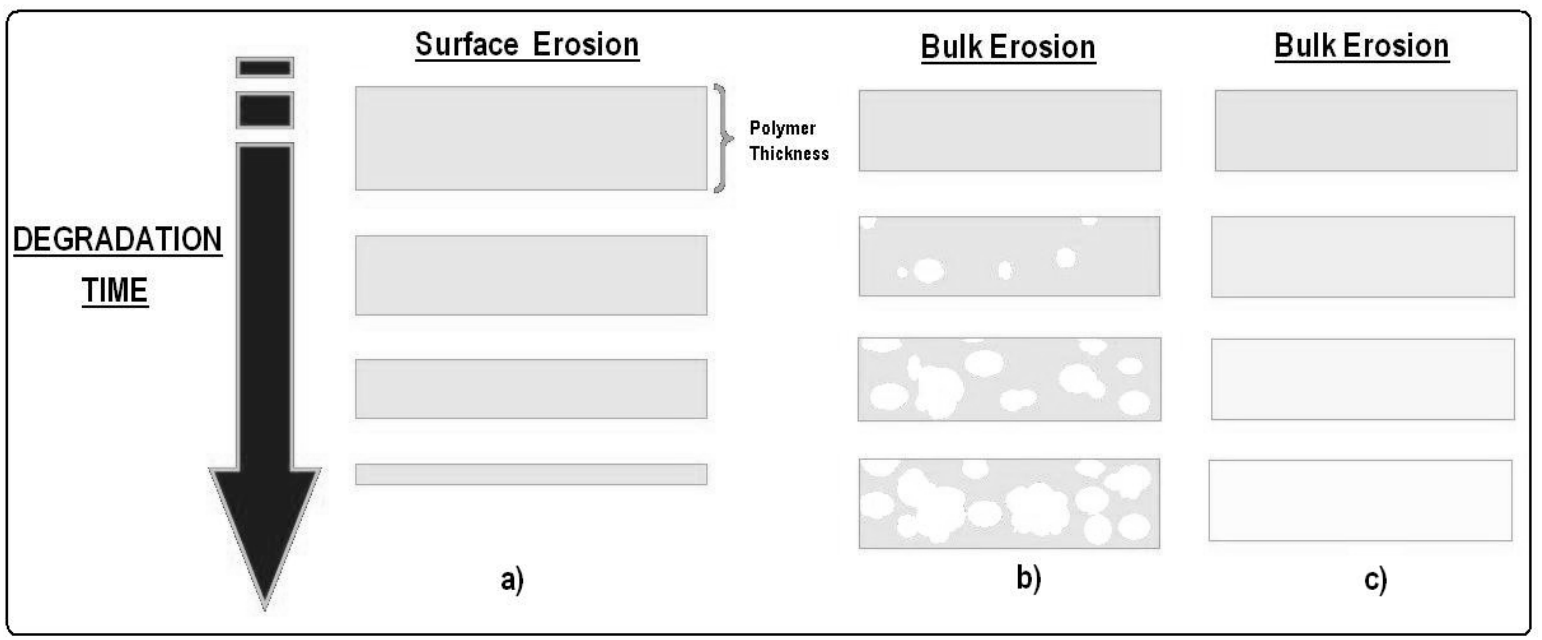

Figure 2 - Schematic illustration of three types of erosion phenomenon:

(a) surface erosion, (b) bulk erosion with autocatalysis and (c) bulk erosion without autocatalysis

Surface and bulk erosion are ideal cases to which most polymers cannot be unequivocally assigned. We can define the characteristic time of hydrolysis, as the inverse of degradation rate:

$$
\tau_{H}=\frac{1}{k E w}=\frac{1}{u_{m}}
$$

If $D$ is the diffusion coefficient of water in the polymer and $L$ is the sample thickness, we can define a characteristic time of diffusion, $\tau_{D}$ :

$$
\tau_{D}=\frac{L^{2}}{D}
$$

When $\tau_{H} \gg \tau_{D}$, water reaches the core of the material before it reacts, and the degradation starts homogenously. This is the case of PLA-PCL (Saha and Tsuji, 2009; Auras, R. et al., 2004). When $\tau_{H} \ll \tau_{D}$, water reacts totally in the superficial layer and will never reach the core of the material. The degradation starts heterogeneously through the volume. In these cases, a higher surface to volume ratio induces a faster degradation. In the presented case, as 
will be shown below, there is no difference in the degradation rate between fibers of different dimensions, meaning that for this range of dimensions and on this conditions of temperature and environment, erosion occurs homogenously.

5. One factor that complicates the erosion of PLA is that the hydrolysis reaction is autocatalytic (Siparsky et al., 1998). For example, a thick plate of PLA erodes faster than a thinner one made of the same polymer (Grizzi et al., 1995). This occurs due to retention of the oligomeric hydrolysis products within the material, which are carboxylic acids, causing a local decrease in $\mathrm{pH}$ and therefore accelerating the degradation (Göpferich, 1996). As can be seen in figure 2 b), hollow structures are formed as a consequence (Grizzi et al., 1995). A more complex model, with more parameters, is necessary to describe this phenomenon. This implies an extensive experimental characterization, which is out of the scope of this work. The hollow formation occurs in the late stages of erosion, when molecular weight becomes highly reduced. The present models, to describe strength decrease and stress-strain plot evolution during degradation, are only valid for the initial phase of erosion, i.e. about 8 weeks. This is the time scale where strength and molecular weight suffer the most significant decrease, as can be seen in results. Hence the present mechanical models neglect the hollow formation effect, since this phenomenon may be neglected during the first 8 weeks, i.e. the mass loss and oligomers diffusion are neglected. Material

\section{Constitutive Models}

A constitutive model is a relationship between the mechanical response of a body and the stress it is subjected to and correspondingly the forces that cause such response. A wide variety of material behaviors are described with a few different classes of constitutive equations. Due to the nonlinear nature of the stress vs. strain plot, the classical linear elastic model is clearly not valid for large deformations. Hence, given the nature of PLA-PCL, classical models such as the neo-Hookean and Mooney-Rivlin models for incompressible hyperelastic materials may be used to describe its mechanical behavior until rupture. For these materials, stiffness depends on the fiber stretch. Mechanical properties of elastomeric materials are usually represented in terms of a strain energy density function $W$. $W$ is a scalar function of the deformation gradient. $W$ can also be represented as a function of the right Cauchy-Green deformation tensor invariants. In general, the strain energy density for an isotropic, incompressible, hyperelastic material is determined by two invariants. The first and second invariants in uniaxial tension are given by:

$$
\begin{aligned}
& I_{C}=\lambda^{2}+\frac{2}{\lambda} \\
& I I_{C}=\frac{1}{\lambda^{2}}+2 \lambda
\end{aligned}
$$

where $\lambda$ is the axial stretch $(\lambda=1+\varepsilon)$, that satisfies $\lambda \geq 1$. The neo-Hookean incompressible hyperelastic solid is given a stored energy function of the form:

$$
W=\frac{\mu_{1}}{2}\left(I_{C}-3\right)
$$


where $\mu_{1}>0$ is the material property, usually called the shear modulus. An extension of this model is the Mooney-Rivlin incompressible hyperelastic solid, which stored energy function has the form:

$$
W=\frac{\mu_{1}}{2}\left(I_{C}-3\right)+\frac{\mu_{2}}{2}\left(I I_{C}-3\right)
$$

with two material properties $\mu_{1}$ and $\mu_{2}$. Higher order stored energy functions may be considered to describe the experimental data, such as a reduced $2^{\text {nd }}$ order stored energy function, that includes a mixed term with both invariants of the right Cauchy-Green stretch tensor and an extra material constant $\mu_{3}$, which stored energy function has the form:

$$
W=\frac{\mu_{1}}{2}\left(I_{C}-3\right)+\frac{\mu_{2}}{2}\left(I I_{C}-3\right)+\frac{\mu_{3}}{6}\left(I_{C}-3\right)\left(I I_{C}-3\right)
$$

The axial nominal stress for the three models, neo-Hookean $\left(\sigma^{N H}\right)$, Mooney-Rivlin $\left(\sigma^{M R}\right)$ and reduced second order $\left(\sigma^{2 n d r e d}\right)$, will be given by:

$$
\begin{aligned}
& \sigma^{N H}=\mu_{1}\left(\lambda-\frac{1}{\lambda^{2}}\right) \\
& \sigma^{M R}=\mu_{1}\left(\lambda-\frac{1}{\lambda^{2}}\right)+\mu_{2}\left(1-\frac{1}{\lambda^{3}}\right) \\
& \sigma^{2^{n d} r e d}=\left(\mu_{1}-\mu_{3}\right)\left(\lambda-\frac{1}{\lambda^{2}}\right)+\left(\mu_{2}-\mu_{3}\right)\left(1-\frac{1}{\lambda^{3}}\right)+\mu_{3}\left(\lambda^{2}-\frac{1}{\lambda^{4}}\right)
\end{aligned}
$$

According to Soares et al. (2010) the model constitutive material parameters depend on degradation time. The material parameters are considered to be material functions of degradation damage instead of material constants. Due the unavailability of experimental data, a simple linear variation from values that characterize the non-degraded response towards values that characterize the full degraded material response was considered in the work of Soares et al. (2010).

\section{Material and Methods}

Suture fibers of PLA-PCL (composition 90:10, initial number average molecular weight $\mathrm{Mn}_{0}=28000$, polydispersion of 3.3 , glass transition temperature $\mathrm{Tg}=56^{\circ} \mathrm{C}$ and melting temperature $\left.\mathrm{Tm}=157^{\circ} \mathrm{C}\right)$, with two different diameters $(150 \mu \mathrm{m}$ and $400 \mu \mathrm{m})$ were provided by Chirmax. Three test pieces, each of $100 \mathrm{~mm}$ long, were cut. Sterile fiber specimens were then placed in $50 \mathrm{ml}$ test tubes and submitted to six different degradation steps under phosphate buffer solution (PBS), at constant temperature $\left(37^{\circ} \mathrm{C}\right)$. This procedure took place in a biological safety cabinet using sterile tools, to assure a sterile degradation. The duration of each stage was previously determined, according to the supplier durability claims, until a maximum of 7 months. Dry weight $\left(24 \mathrm{~h}\right.$ in incubator at $\left.37^{\circ} \mathrm{C}\right)$ was measured initially and at the end of each stage, to determine material erosion.

The number-average molecular weight was determined at the end of each step, by GPC Polymer PL50, using a Polymer ResiPore column $(300 \times 7.5 \mathrm{~mm})$, and chloroform as solvent and eluent. The sample of around $2.5 \mathrm{mg}$ of degraded fiber was dissolved in $1 \mathrm{ml}$ of chloroform and placed in the ultra-sounds during $5 \mathrm{~min}$. After filtration the sample was tested. 
Mechanical properties were also evaluated at the end of each stage. A universal mechanical test machine (TIRAtest 2705) was used, with a load cell off $100 \mathrm{~N}$, and pneumatic grips commonly used in fiber testing. The distance between grips was set for 50 $\mathrm{mm}$. Three specimens were used for each fiber sample. The displacement rate was $500 \mathrm{~mm} / \mathrm{min}$. The deformation energy was calculated by the integral of the nominal stressstrain curve. The constitutive parameters for hyperelastic constitutive models were then defined based on these uniaxial data, for each degradation step.

\section{Results and Discussion}

As seen in figure 3, after 28 weeks of degradation, PLA-PCL fibers had lost about $20 \%$ of its weight and no significant changes were observed among dimensions and degradation media. The initial $\mathrm{pH}$ of the PBS solution was 8 and did not change significantly during degradation.

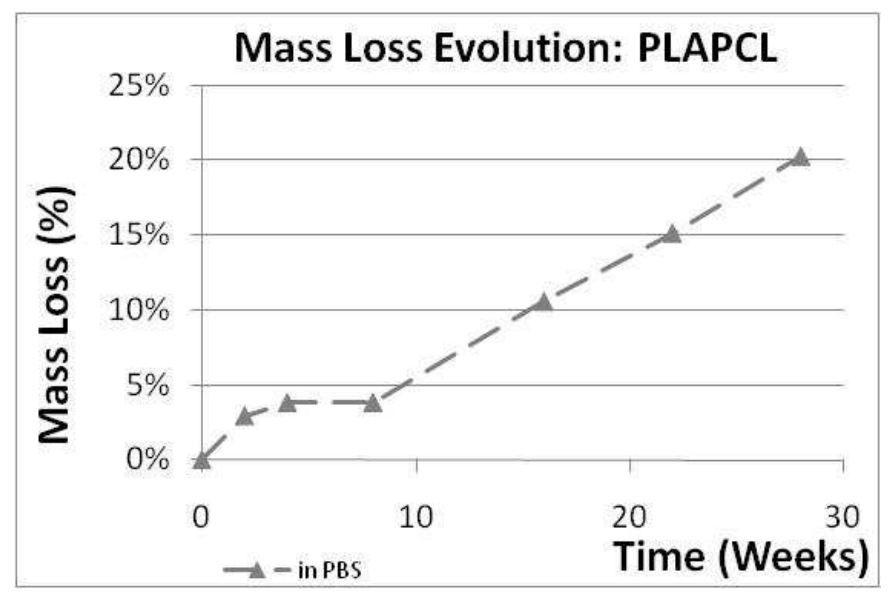

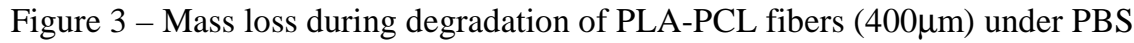

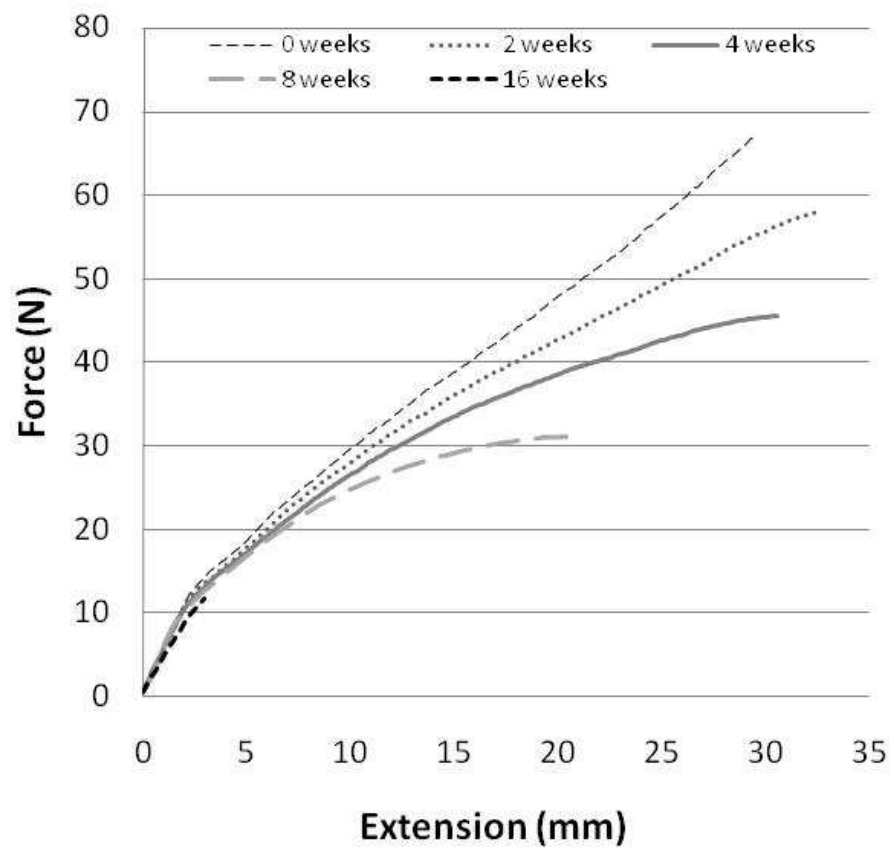

Figure 4 - Tensile test results during degradation of PLA-PCL fibers $(400 \mu \mathrm{m})$ under PBS 
As can be seen in figure 4, PLA-PCL has become brittle only after 16 weeks, lost its plasticity stage, and strength has progressively decreased. The almost constant slope of the linear elastic stage indicates that no significant variation in Young modulus occurred during degradation. These results are in accordance with those of Tsuji et al. (1998) for blends of PLA-PCL.

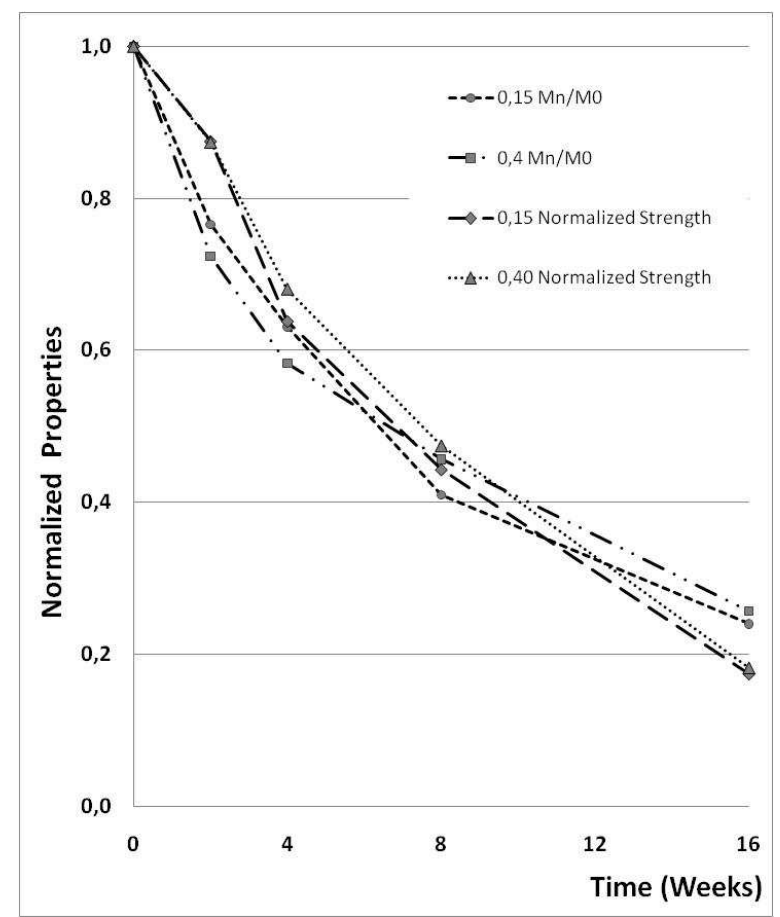

Figure 5 - Normalized: a) strength and b) molecular weight, during degradation of PLA-PCL fibers, of $150 \mu \mathrm{m}$ and $400 \mu \mathrm{m}$, under PBS

While in the first 16 weeks the fiber only looses $10 \%$ of mass, it presents $80 \%$ of strength loss. For these PLA-PCL fibers, no significant differences were observed among the different dimensions tested, either in terms of strength and molecular weight evolutions during degradation (see figure 5). One can conclude that, in the present case, water diffusion can be assumed instantaneous and that hydrolysis takes place homogenously throughout the samples (bulk degradation without autocatalysis) (Auras, R. et al., 2004). 


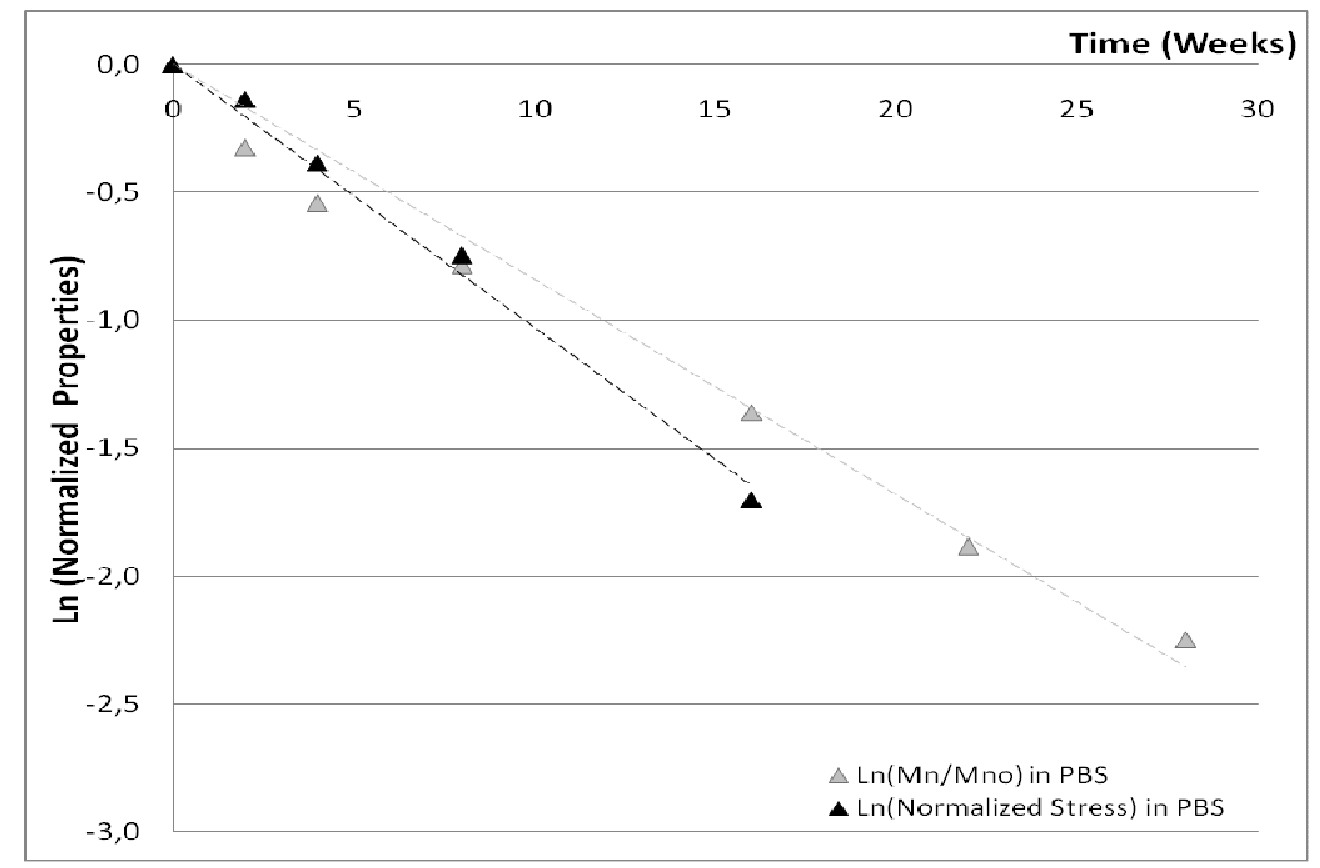

Figure 6 - Normalized strength and normalized molecular weight evolution during degradation of PLA-PCL fibers of $400 \mu \mathrm{m}$ under PBS

From figure 6, one can see that the measured strength follows the same trend as the molecular weight, in a semi-logarithmic representation. Instead of equation 6, a relationship similar to the one obtained for the molecular weight, equation 5, can be used,

$$
\sigma=\sigma_{0} e^{-u_{s} t}
$$

where $u_{s}$ is the strength decrease rate of the material. This parameter, $u_{s}$, seems to be directly related to the molecular weight decrease rate of the material, $u_{m}$, as can be seen in figure 6 and in table 1. This same trend can be found in the degradation results of other previous works, such as the one by Meek et al. (2004), with PDLA-PCL. This can therefore provide a strategy to obtain a design failure criteria for the evolution of the limit strength of the device during the degradation process, $\sigma=f(t)$.

Table 1 - Degradation rates of tensile strength, $\boldsymbol{u}_{s}$, and molecular weight, $\boldsymbol{u}_{\boldsymbol{m}}$, under PBS

\begin{tabular}{ccccc}
\hline & $\operatorname{Ln}\left(\sigma / \sigma_{o}\right)=-u_{s} t$ & & $\operatorname{Ln}\left(M_{n} / M_{o}\right)=-u_{m} t$ \\
& $\boldsymbol{u}_{\boldsymbol{s}}$ & $R$ & $\boldsymbol{u}_{\boldsymbol{m}}$ & $R$ \\
\hline $\boldsymbol{P B S}$ & 0,103 & 0,996 & 0,0841 & 0,989 \\
\hline
\end{tabular}

The hydrolytic damage (eq. 7) can be rewritten in the form:

$$
d_{\mathrm{h}}=1-\frac{\sigma}{\sigma_{0}}=1-e^{-u_{s} t}=1-e^{-k E w t}
$$

So the hydrolytic damage depends on the hydrolysis kinetic constant, $k$, the concentrations of ester groups, $E$, the water concentration in the polymer matrix, $w$, and the 
degradation time. In this example, we assume that the degradation rate, $u$, is constant, and damage only depends on degradation time. But as it was shown previously, in other cases it may not be this way. For highly heterogeneous erosion the rate will not be constant, and the water concentration will depend on the position and time.

From figure 7, one can see that the hyperelastic material models fit well the measured storage energy, for all the degradation steps up to 16 weeks. The experimental data of storage energy was calculated by measuring the area (i.e., by taking the integral) underneath the stress-strain curve, from zero until a certain level of stretch. The neo-Hookean model is the less precise. Only one material model parameter, $\mu_{1}$, changes during degradation for the three material models, as can be seen in table 2 . However the constitutive models are unable to describe precisely the initial elastic phase of the stress-strain plot, were the stiffness remains barely constant. This explains why the material model parameter, $\mu_{1}$, increases sharply in the last degradation stage (16 weeks), because the inverse parameterization was based on the experimental data that mostly has elastic deformation. If this last degradation stage is omitted, one can see that the material model parameter, $\mu_{1}$, varies linearly with the hydrolytic damage, as proposed by Soares et al. (2010). 

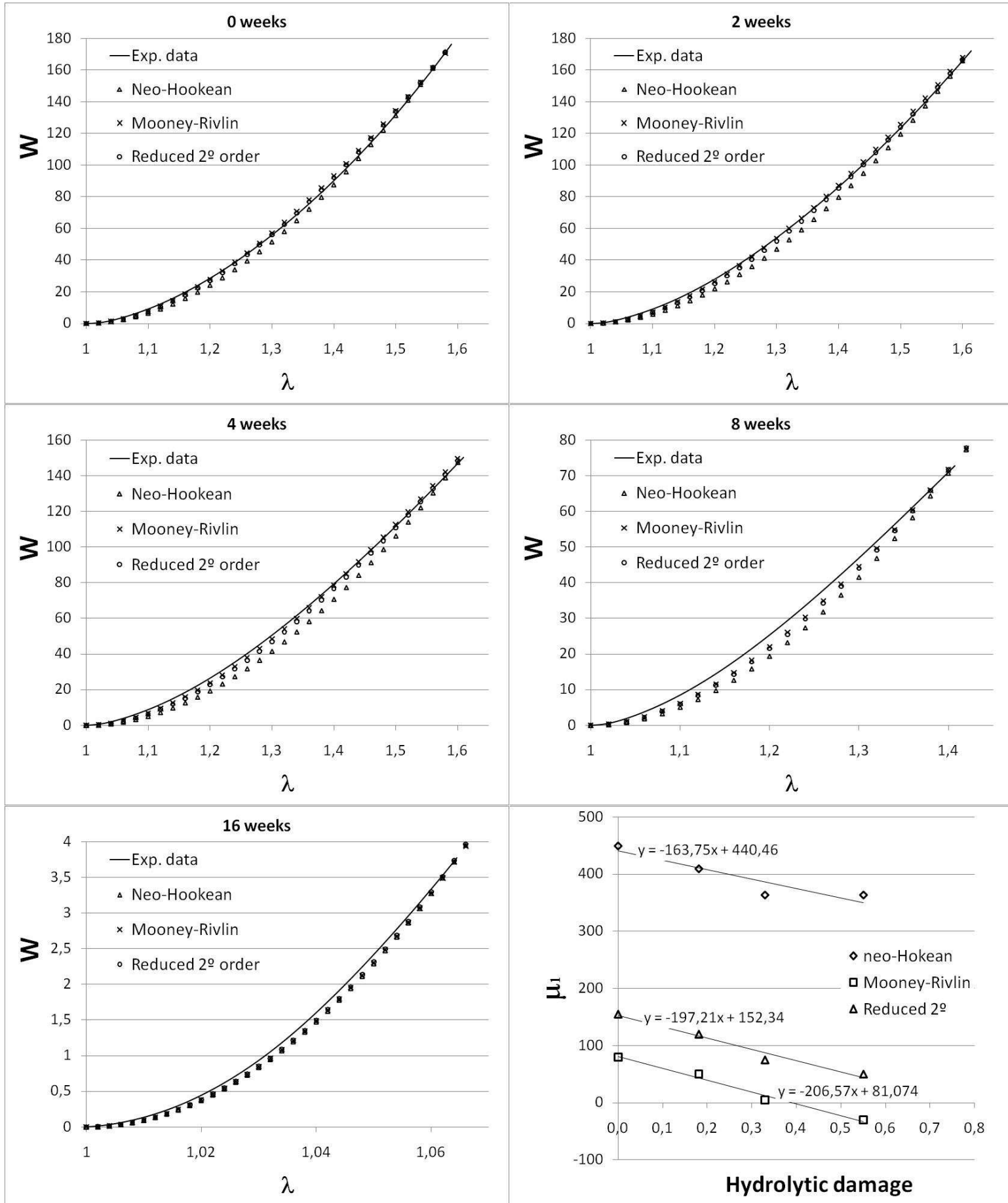

Figure 7 -Storage energy vs. axial stretch for $0,2,4,8$ and 16 weeks of degradation, and evolution of the material parameter, $\mu_{l}$, of the models during degradation 


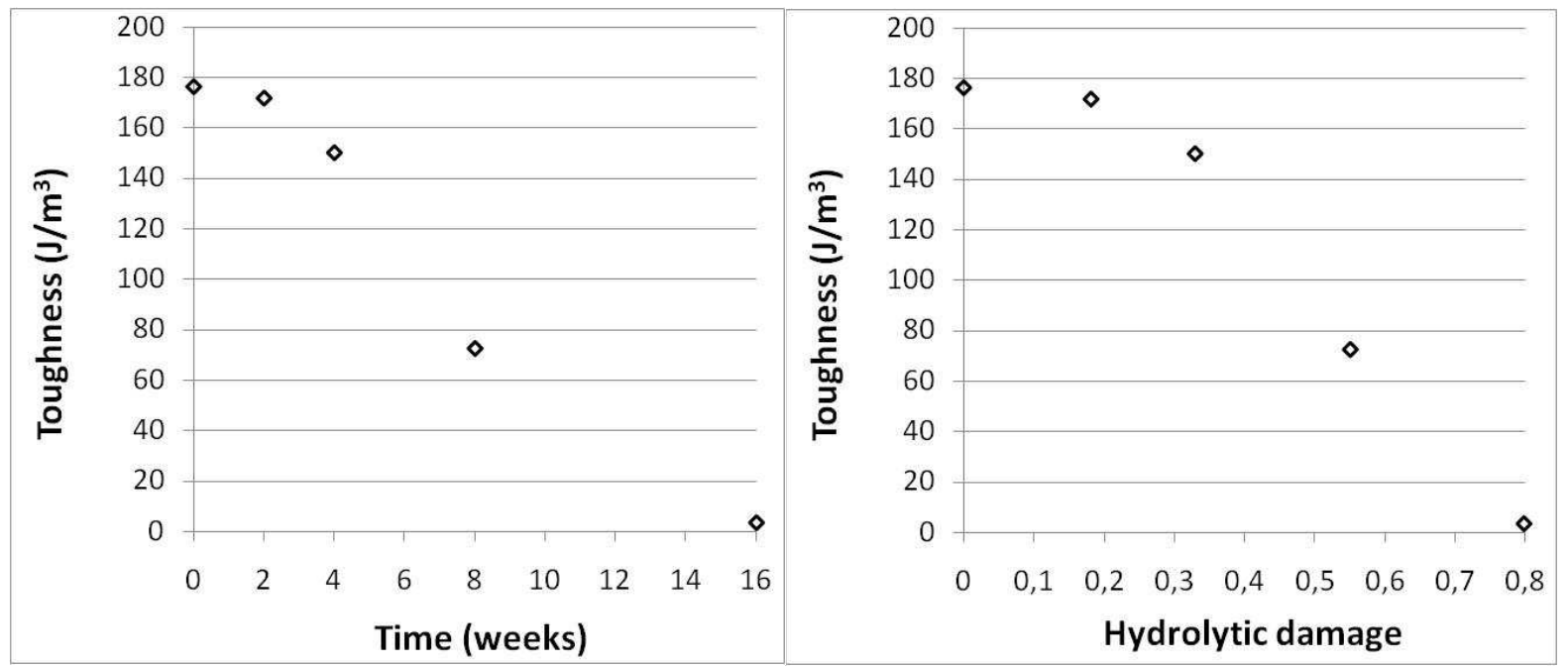

Figure 8 - Evolution of toughness with: a) degradation time and b) hydrolytic damage

In figure 8 , the evolution of maximum storage energy, or the toughness of the material during degradation is presented. This depends on the maximum stress that can be calculated from eq. 18, and the material behavior that can be estimated by one of the constitutive models presented here (equations 15, 16 and 17).

Table 2 - Evolution of the models material parameters during degradation

\begin{tabular}{lccccc}
\hline Material Models & weeks & $\mathbf{d}$ & $\boldsymbol{\mu}_{\mathbf{1}}$ & $\boldsymbol{\mu}_{\mathbf{2}}$ & $\boldsymbol{\mu}_{\mathbf{3}}$ \\
\hline & 0 & 0.00 & 450 & & \\
Neo-Hookean & 2 & 0.18 & 410 & & - \\
& 4 & 0.33 & 364 & - & \\
& 8 & 0.55 & 364 & & \\
Mooney-Rivlin & 16 & 0.80 & 630 & & \\
& 0 & 0.00 & 80 & & \\
& 2 & 0.18 & 50 & & \\
& 4 & 0.33 & 5 & 500 & \\
& 8 & 0.55 & -30 & & \\
$2^{\text {nd }}$ reduced order & 16 & 0.80 & 150 & & \\
& 0 & 0.00 & 155 & & \\
& 2 & 0.18 & 120 & & \\
& 4 & 0.33 & 75 & 400 & \\
\hline
\end{tabular}

From figure 9, one can see that the hyperelastic material models allowed a reasonable approximation of the tensile test results. The presented method, that consists on changing the first material parameter with hydrolytic damage, $\mu_{1}(d)$, according to the linear regression presented in figure 7 , enables to describe the mechanical behavior evolution by using equations 15,16 or 17 , while the limit stress is defined by equation 18 . As one can see in figure 9, the models are less precise for damage over 50\% (more than 8 weeks). However, this validity limit of the models may be higher for swollen specimens. 

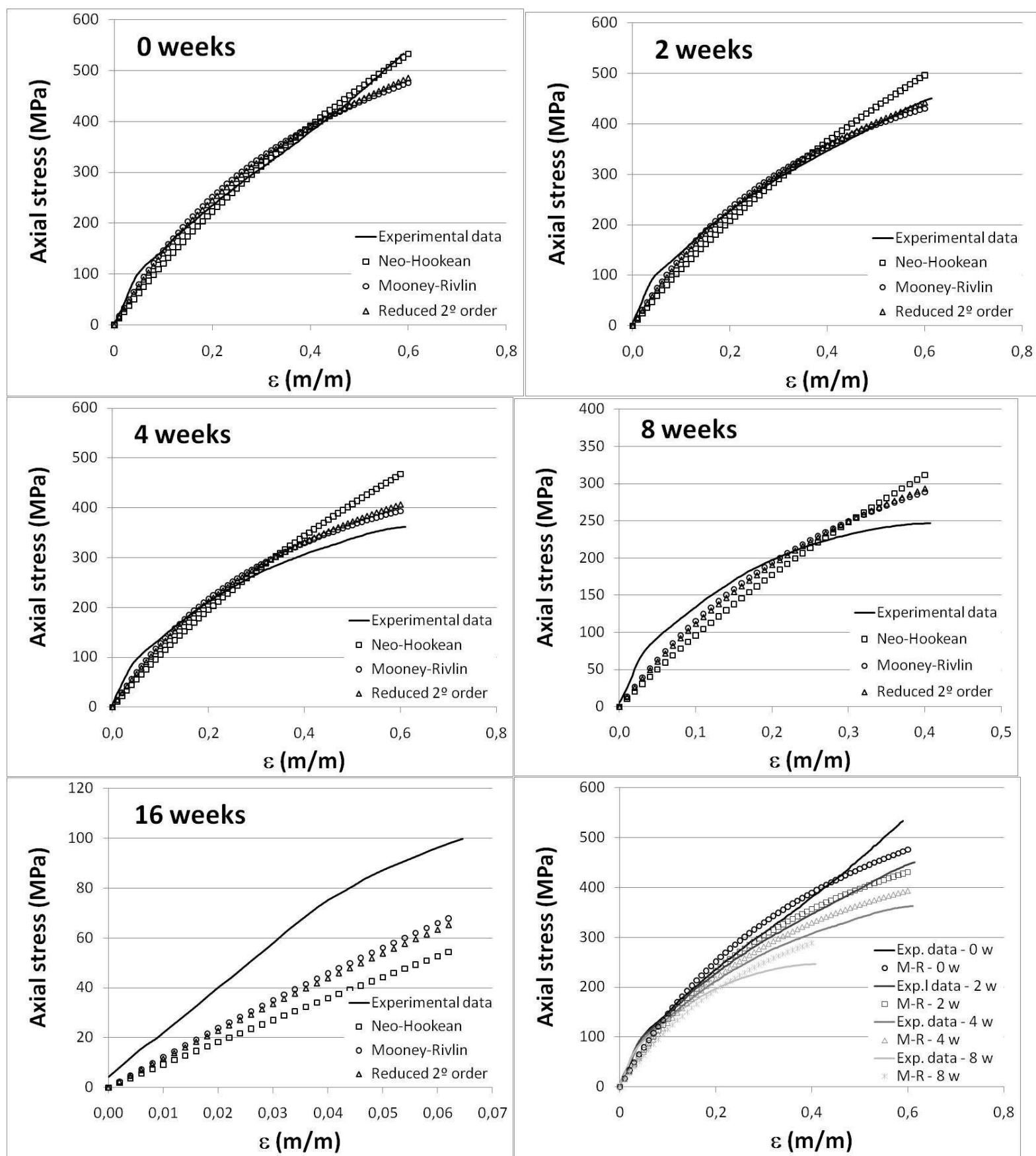

Figure 9 -Axial nominal stress vs. strain for 0,2, 4, 8 and 16 weeks of degradation (experimental data and material models results) and tensile test results during degradation with corresponding results of Mooney-Rivlin material model.

\section{Conclusions}

Tensile strength evolution can be determined during degradation in test specimens of PLA-PCL fibers or other thin elements. This is possible since hydrolytic reaction is the limiting step of the overall degradation process. Diffusion may be neglected in these cases, and hydrolysis may be considered to take place homogeneously within the sample volume. The degradation rate can be further used as a failure criterion in the dimensioning of biodegradable devices, using a first order kinetic equation. From the processed results of Hill 
(1977), we observed that the time dependence of the tensile strength during degradation process is similar to the one exhibited by the molecular weight. When loading conditions are simple and the desired time of mechanical support is known, a "trial and error" approach may be sufficient to design reasonable reliable devices. In more complex situations, device designers can use numerical approaches to define the material formulation and geometry that will satisfy the immediate needs of symptomatic relief. However, the lack of design tools to predict long term behavior has limited the application of biodegradable materials.

The simple modeling technique presented here allows pre-clinical evaluation of the functional compatibility and the optimization by comparison of different solutions in terms of long-term biomechanical behavior. These constitutive models may be implemented in commercial finite element software packages like ABAQUS, by changing the material parameter as function of damage, and associated to the failure criterion implemented by a user defined (UMAT) subroutine. These can also be applied to more complicated numerical models in 3D applications. The simple method presented here, only valid for low thickness devices in the first steps of erosion, and without considering the degradation rate dependence on temperature, environment and stress state, can be further updated to more detailed models that consider these dependencies, the crystalline degree dependence, and the diffusion of water, enzymes and degradation products. In these further complicated problems, damage will depend not only on the degradation time, but also on the water concentration and the hydrolysis kinetic constant, no longer constants but time, geometrical, degradation media, temperature and stress state dependents.

\section{Acknowledgments}

The authors would like to thank FCT for financial support under the grant PTDC/EMEPME/70155/2006 and CHIRMAX for material supply. João Ferra wishes to thank FCT for the $\mathrm{PhD}$ grant SFRH/BD/23978/2005. André Vieira wishes to thank FCT for the PhD grant SFRH/BD/41195/2007.

\section{References}

Auras, R., Harte, B., Selke, S., 2004. An Overview of Polylactides as Packaging Materials. Macromolecular Bioscience. 4, 835-864.

Bellenger, V., Ganem, M., Mortaigne, B., Verdu, J., 1995. Lifetime prediction in the hydrolytic ageing of polyesters. Polymer Degradation and Stability. 49, 91-97.

Bikiaris, D., Papageorgiou, G., Achilias, D., Pavlidou, E., Stergiou, A., 2007. Miscibility and enzymatic degradation studies of poly(e-caprolactone)/poly(propylene succinate) blends. European Polymer Journal. 43, 2491-2503.

Chen, C.C., Chueh, J.Y., Tseng, H., Huang, H.M., Lee, S.Y., 2003. Preparation and characterization of biodegradable PLA polymeric blends. Biomaterials. 24, 1167-1173.

Cheung, H.Y., Lau, K.T., Lu, T.P., Hui, D., 2007. A critical review on polymer-based bio-engineered materials for scaffold development. Composites: Part B. 38, 291-300.

Chu, C.C., 1985. Strain-accelerated hydrolytic degradation of synthetic absorbable sutures. In: C.W. Hall (Eds.), Surgical research, recent developments: Proceedings of the First Annual Scientific Session of the Academy of Surgical Research (edition, pp. 111-115). San Antonio, Pergamon Press.

Crank, J., 1975. The mathematics of diffusion. 2nd ed. Polymer, Issue 11, ed. G.S. Park. Vol. Volume 16. Oxford: Clarendon Press. 855. 
Farrar, D.F., Gilson, R.K., 2002. Hydrolytic degradation of polyglyconate B: the relationship between degradation time, strength and molecular weight. Biomaterials. 23, 3905-3912.

Gan, Z., Yu, D., Zhong, Z., Liang, Q., Jing, X., 1999. Enzymatic degradation of poly(«-caprolactone)/poly(dllactide) blends in phosphate buffer solution. Polymer. 40, 2859-2862.

Göpferich, A., Langer, R., 1993. Modeling of Polymer Erosion. Macromolecules. 26, 4105-4112.

Göpferich, A., 1996. Mechanisms of polymer degradation and erosion. Biomaterials. 17, 103-114.

Grizzi, I., Garreau, H., Li, S., Vert, M., 1995. Hydrolytic degradation of devices based on poly [DL-lactic acid] size dependence. Biomaterials. 16, 305-311.

Han, X., Pan, J., 2009. A model for simultaneous crystallization and biodegradation of biodegradable polymers. Biomaterials. 30,423-430.

Hakkarainen, M., Karlsson, S., Albertsson, A-C., 2000. Rapid (bio)degradation of polylactide by mixed culture of compost microorganisms-low molecular weight products and matrix changes. Polymer. 41, 2331-2338.

Hill, C.R., 1977. An Introduction to Chemical Engineering Kinetics and Reactor Design. New York. John Wiley \& Sons.

Kirby, A.J., 1972. Hydrolysis and formation of esters of organic acids. In: Bamford CH, Tipper CFH, eds. Comprehensive Chemical Kinetics (Vol 10: Ester Formation and Hydrolysis and Related Reactions).

Amsterdam: Elsevier, 57-202.

Li, S., Garreau, H., Vert, M., 1990. Structure-property relationships in the case of the degradation of massive aliphatic poly( $\alpha$-hydroxyacids) in aqueous media. Part 2: degradation of lactide/glycolide copolymers: PLA37.5GA25. and PLA75GA25. J Mater Sci Mater Med. 1, 131-139.

Liao, S., Chan, C.K., Ramakrishna, S., 2008. Stems Cells and Biomimetic Materials Strategies for Tissue Engineering. Materials Science and Engineering C. 28, 1189-1202.

Meek, M., Jansen, K., Steendam, R., van Oeveren, W., van Wachem, P., van Luyn, M., 2004. In vitro degradation and biocompatibility of poly(DL-lactide- $\varepsilon$-caprolactone) nerve guides. J. Biomed. Mater. Res. A. 68(1), 43-51.

Metzmacher, I., Radu, F., Bause, M., Knabner, P., Friess, W., 2007. European Journal of Pharmaceutics and Biopharmaceutics. 67, 349-360.

Miller, N.D., Williams, D.F., 1984. The in vivo and in vitro degradation of poly(glycolic acid) suture material as a function of applied strain. Biomaterials. 5, 365- 368.

Nair, L.S., Laurencin, C.T., 2007. Biodegradable polymers as biomaterials. Prog Polym Sci. 32, 762-798.

Park, K., Shalaby.W., Park, H., 1993. Biodegradable Hydrogels for Drug Delivery. Lancaster: Technomic Publ.

Saha S. K., Tsuji H., 2009. Enhanced Crystallization of Poly(L-lactide-co-ecaprolactone) in the Presence of Water. J Appl Polym Sci. 112, 715-720.

Siparsky, G.L., Voorhees, K.J., Miao, F., 1998. Hydrolysis of polylactic acid (PLA) and polycaprolactone (PCL) in aqueous acetonitrile solutions: autocatalysis. Journal of Environmental Polymer Degradation. 6, 3141.

Sykes, P., 1975. A Guidebook to Mechanism in Organic Chemistry 4th ed. London, UK. Longman Group Ltd.

Soares, J. S., Rajagopal, K. R., Moore, J. E., 2010. Deformation-induced hydrolysis of a degradable polymeric cylindrical annulus. Biomech Model Mechaobiol. 9, 177-186.

Todo, M., Park, S.-D., Takayama, T., Arakawa, K., 2007. Fracture micromechanisms of bioabsorbable PLLA/PCL polymer blends. Engineering Fracture Mechanics. 74, 1872-1883.

Tsuji, H., Ikada, Y., 1998. Properties and morphology of poly(L-lactide) - II hydrolysis in alkaline solution. J. Polym. Sci., Part A: Polym. Chem. 36, 59-66. 
Tsuji, H., Ikada, Y., 2000. Properties and morphology of poly(L-lactide) 4. Effects of structural parameters on long-term hydrolysis of poly(L-lactide) in phosphate-buffered solution. Polym. Degrad. Stab., 67, 179-189.

Tsuji, H., Mizuno, A., Ikada, Y., 1998. Blends of Aliphatic Polyesters. III. Biodegradation of Solution-Cast Blends from Poly(L-lactide) and Poly(«-caprolactone). J. Appl. Polym. Sci. 70, 2259-2268.

Tsuji, H., Nakahara, K., 2001. Poly(L-lactide) - IX Hydrolysis in acid media. J. Appl. Polym. Sci. 86, $186-194$.

Yu, R., Chen, H., Chen, T., Zhou, X., 2008. Modeling and simulation of drug release from multi-layer biodegradable polymer microstructure in three dimensions. Simulation Modelling Practice and Theory. 16, 1525.

Van de Velde, K.,. Kiekens, P., 2002. Biopolymers: overview of several properties and consequences on their applications. Polymer Testing. 21, 433-442.

Vieira, A., Guedes R. , Marques A., 2009. Development of Ligament Tissue Biodegradable Devices: A Review, J. Biomechanics. 42 (15), 2421-30.

Wang Y., Pan, J., Han, X., Sinka, C., Ding, L., 2008. A phenomenological model for the degradation of biodegradable polymers. Biomaterials. 29, 3393-3401.

Ward, I., 1983. Mechanical properties of solid polymers, 2nd ed. Chichester, UK. Wiley \& Sons.

Williams, D.F., 1981. Enzymatic hydrolysis of polylactic acid. Engng Med. 10(1), 5-7.

Zhang, X., Espiritu, M., Bilyk, A., Kurniawan, L., 2008. Morphological behaviour of poly(lactic acid) during hydrolytic degradation Polymer Degradation and Stability. 93, 1964-1970.

Zuideveld, M., Gottschalk, C., Kropfinger, H., Thomann, R., Rusu, M., Frey, H., 2006. Miscibility and properties of linear poly(L-lactide)/branched poly(L-lactide) copolyester blends. Polymer. 47, 3740-3746. 